\title{
Anteroposterior diameter of the infrarenal abdominal aorta is higher in women with polycystic ovary syndrome
}

This article was published in the following Dove Press journal:

Vascular Health and Risk Management

23 June 2009

Number of times this article has been viewed

\author{
Marco Matteo Ciccone' \\ Stefano Favale' \\ Anish Bhuva ${ }^{4}$ \\ Pietro Scicchitano' \\ Vito Caragnano' \\ Cristina Lavopa ${ }^{2}$ \\ Giovanni De Pergola ${ }^{3}$ \\ Giuseppe Loverro \\ 'Department of Cardiology; \\ ${ }^{2}$ Department of Gynecology \\ and Obstetrics; ${ }^{3}$ Section of Internal \\ Medicine, Endocrinology, Andrology, \\ and Metabolic Diseases, University \\ of Bari, DETO, Italy; ${ }^{4}$ University \\ College of London, London, UK
}

Background: Women affected by polycystic ovary syndrome (PCOS) are known to be at higher risk of cardiovascular disease. The aim of this study was to identify the artery that first is affected by early pre-atherosclerotic changes in PCOS.

Methods: Twenty-nine women with PCOS aged 17 to 27 years and 26 healthy nonhyperandrogenic volunteers with regular menses (control women) aged 16 to 28 years were enrolled. All PCOS patients were overweight or obese (body mass index $[\mathrm{BMI}] \geq 25$ ). Diagnosis of PCOS was performed in line with the 2003 Rotterdam ESHRE/ASRM-Sponsored PCOS Consensus Workshop Group. Accordingly, PCOS was defined when at least two of the following three features were present after exclusion of other etiologies: 1) oligomenorrhea and or anovulation; 2) hyperandrogenism and/or hyperandrogenemia; and 3) polycystic ovaries visible at ultrasound. Androgen excess or related disorders were excluded. The intima-media thickness (IMT) of common carotid arteries and common femoral arteries and the anteroposterior diameter of the infrarenal abdominal aorta were measured by ultrasound. Lutenizing hormone $(\mathrm{LH})$, follicle-stimulating hormone (FSH), estradiol, total testosterone, androstenedione, and sex hormone-binding globulin (SHBG) serum levels were measured between the $3 \mathrm{rd}$ and the 6 th day of spontaneous or progestin-induced menstrual cycle. Our study was performed in the absence of any medical treatment.

Results: Women with PCOS showed a higher LH to FSH ratio $(\mathrm{p}<0.01)$, increased fasting insulin ( $\mathrm{p}<0.001)$, total testosterone $(\mathrm{p}<0.001)$, and androstenedione $(\mathrm{p}<0.001)$ levels, and lower SHBG concentrations ( $\mathrm{p}<0.001$ ) compared to control women. BMI and waist-to-hip ratio were also higher in women with $\operatorname{PCOS}(\mathrm{p}<0.000$ and $\mathrm{p}<0.001$, respectively). Women with PCOS also showed increased total cholesterol $(p<0.001)$, triglyceride $(p<0.001)$, and apolipoprotein B $(\mathrm{p}<0.001)$ levels. Vascular data showed women with PCOS had a higher anteroposterior diameter than control women $(p<0.005)$. However, when analysis of covariance was performed and BMI was entered into the model as a covariate, anteroposterior diameter did not maintain a significant association with PCOS.

Conclusion: This study shows that anteroposterior diameter of the infrarenal abdominal aorta, but not IMT of common carotid arteries or common femoral arteries, is higher in women with PCOS than in women without this disease. This represents the earliest atherosclerotic change in women with PCOS. However, this alteration seems to be due to body weight secondary to PCOS and not due to PCOS per se.

Keywords: polycystic ovary syndrome, antero-posterior diameter, infrarenal abdominal aorta, intimia-media thickness

\section{Introduction}

Polycystic ovary syndrome (PCOS) is a clinical association of anovulation and hyperandrogenism, but it is often associated with irregular menses, infertility, acne,
Correspondence: Marco Matteo Ciccone Institute of Cardiovascular Diseases, Azienda Ospedaliera Policlinico, Piazza Giulio Cesare II, 70 I24-I, Italy

Tel +39080 5478627

Fax +39080 5478627

Email ciccone@cardio.uniba.it 
and hirsutism. ${ }^{1-4}$ Although it is important to recognize and address these clinical problems, attention has also turned to the risk of diabetes and cardiovascular disease $\mathrm{e}^{5}$ due to central obesity, ${ }^{6,7}$ dyslipidemia (low high-density lipoprotein [HDL] and higher cholesterol and triglyceride levels), ${ }^{6,8}$ hypertension, ${ }^{6}$ endothelial dysfunction, ${ }^{9}$ and insulin resistance. ${ }^{10,11}$

Shaw and colleagues recently reported that women with clinical features of PCOS have five years lower cardiovascular event-free survival than women without clinical features of PCOS. ${ }^{12}$ The mechanisms responsible for higher cardiovascular risk are dyslipidemia, consisting of higher triglycerides, lower HDL cholesterol, and small increases in low-density lipoprotein (LDL) particles, insulin resistance, compensatory hyperinsulinemia, central obesity, and androgen excess. ${ }^{13,14}$

Vascular preclinical abnormalities have been recently demonstrated in women with PCOS by using Doppler ultrasound. In particular, several studies found an increased intima-media thickness (IMT) of common carotid artery in PCOS patients. ${ }^{15-17}$ To the best of our knowledge, there are no data concerning the IMT of common femoral arteries and the anteroposterior diameter of abdominal aorta in women affected by PCOS. Moreover, it is not known which is the earliest alteration in these patients: common carotid artery thickening, common femoral artery thickening, or an enlargement of the anteroposterior diameter of the infrarenal abdominal aorta.

Wilmink and colleagues have shown that the use of ultrasounds to measure the infrarenal aortic diameter is attractive as it is rapid, cheap, and noninvasive. ${ }^{18}$ These characteristics allow serial testing before proceeding with an invasive procedure. A serial scanning can increase the detection rate and decrease the false positive rate. The good accuracy of infrarenal aortic diameter measurements by ultrasound makes this method acceptable for clinical decision-making.

The present study examines whether 1) the IMT of common carotid arteries and common femoral arteries is higher in women with PCOS than in those without this disease (control), 2) the anteroposterior diameter of abdominal aorta is different between the two groups, and 3) to identify in patients with PCOS which is the earliest alteration: common carotid artery thickening, common femoral artery thickening, or enlargement of the anteroposterior diameter of the infrarenal abdominal aorta. Twenty-nine young women affected by PCOS and not taking any drugs were investigated.

\section{Material and methods}

\section{Study population}

Twenty-nine patients with PCOS aged 17 to 27 years were consecutively enrolled in the Department of Obstetrics and
Gynecology, General Hospital Policlinico of Bari, Italy. Diagnosis of PCOS was performed in line with the 2003 Rotterdam ESHRE/ASRM-Sponsored PCOS Consensus Workshop Group. ${ }^{1}$ PCOS was defined when at least two of the following three features were present after exclusion of other etiologies: 1) oligomenorrhea and or anovulation; 2) hyperandrogenism and/or hyperandrogenemia; and 3) polycystic ovaries visible at ultrasound. All patients were overweight or obese (body mass index $[\mathrm{BMI}] \geq 25$ ) and none of them had been treated with hormones in the previous 12 months.

The control group consisted of twenty-six healthy volunteers aged 16 to 28, with regular menses (cycle length, $28.1 \pm 1.3$ days), and normal ultrasonographic appearance of ovaries.

Additional endocrine diseases (hypothyroidism, hyperthyroidism, hyperprolactinemia, Cushing's syndrome, and congenital adrenal hyperplasia screening) were excluded by hormone measurements in all PCOS and control women, and all of them had normal renal and hepatic function.

Hormonal assays, lipid analysis, and ultrasonography were performed in the early follicular phase, between the 3 rd and the 6th day of the menstrual cycle. Menstruation was spontaneous or progestin-induced in women with PCOS, spontaneous in all control women, and progestin-induced in oligomenorrhoic women.

Hirsutism was evaluated according to the Ferriman-Gallwey score in all women and anthropometric parameters obtained by measurement of height, weight, and waist and hip circumferences. BMI was computed as the ratio of weight divided by height squared $\left(\mathrm{kg} / \mathrm{m}^{2}\right)$, while the waist-to-hip ratio (WHR) was calculated as the ratio of waist circumference divided by hip circumference. All women were screened with a standard two-hour oral glucose tolerance test (OGTT). None of the control women showed fasting hyperinsulinemia ( $\geq 15 \mu \mathrm{U} / \mathrm{ml}$ ) or glucose intolerance. Only two women with PCOS showed glucose intolerance and none of them showed diabetes. Blood pressure was recorded on at least three different occasions using a mercury manometer with an appropriate cuff size.

\section{Hormonal data and biochemical evaluation}

Blood samples for hormonal data were drawn between 8.00 AM and 9.00 AM after an overnight fast on the same day of the ultrasound. Serum lutenizing hormone (LH), follicle-stimulating hormone $(\mathrm{FSH})$, estradiol $\left(\mathrm{E}_{2}\right)$, and testosterone were measured by enzyme immunoassay kits 
(Biochemical Immunosystem, Guidonia-Rome, Italy). Androstenedione (DiaSorin GMBH, Dusseldorf, Germany), insulin (Cis Bio International, Vercelli, Italy), and serum sex hormone-binding globulin (SHBG) (Euro-Diagnostica, Malmö, Sweden) were determined by using commercially available radioimmunoassay (RIA) kits. The intraassay and interassay coefficients of variation of all these methods did not exceed $10 \%$ and $15 \%$, respectively. Serum glucose was measured by the glucose oxidase method (Boehringer, Ingelheim, Germany). OGTT was performed by administering $75 \mathrm{~g}$ of D-glucose per os and collecting blood samples before ( 0 minutes), and after 30, 60, 90, and 120 minutes from oral administration.

Fasting lipid profile included the measurement of total cholesterol, HDL cholesterol, LDL cholesterol, triglycerides, apolipoproteins A-1 (apo A-1) and B (apo B) levels. Fasting serum levels of lipids were measured by commercial enzymatic methods (Boehringer Mannheim, Lewes, UK). The lipid determinations were made on the supernatants, but increased assay sensitivity was achieved by increasing the sample volume and adding tribromohydroxybenzoic acid to a concentration of $0.5 \mathrm{~g} / \mathrm{L}$ in the assay reagent. Apo A-1 and apo B were measured by rate immunonephelometry (Beckman Instruments, Fullerton, CA, USA). All these methods showed intraassay and interassay coefficients of variation of $5 \%$ and $9 \%$, respectively.

\section{Transvaginal ultrasound of the ovaries}

In the morning, during the early follicular phase (cycle day 2 , 3 , or 4), a transvaginal ultrasound was performed by means of a transvaginal ultrasound scanner (Toshiba Corp., Tokyo, Japan) with a 5.0 MHz probe. In each ovary, the total number of small follicles ( 2 to $10 \mathrm{~mm}$ ) was counted. Enlarged ovary with multiple small follicles arranged around an echogenic stroma were considered typical ultrasonographic ovarian findings.

\section{Vascular ultrasound studies}

All PCOS and control women underwent high-definition vascular echocardiography to identify the artery that was first affected by early atherosclerosis. High-definition vascular echocardiography was performed in PCOS and control subjects according to the following protocols.

\section{Evaluation of common carotid artery IMT}

Ultrasonographic studies of common carotid arteries were performed bilaterally by a single observer. The value of common carotid arteries that was considered for statistical analyses was the mean of right and left measurements. All studies were performed with a Hewlett Packard Sonos 1500 (Hewlett Packard, Avondale, PA, USA) using a $7.5 \mathrm{MHz}$ high-resolution probe. IMT was defined as a low-level echo grey band that does not project into the arterial lumen. It was measured during end-diastole as the distance from the leading edge of the second echogenic line of the far walls of the distal segment of the common carotid artery, the carotid bifurcation, and the initial tract of internal carotid artery on both sides. Measurements were performed 0.5, 1, and $2 \mathrm{~cm}$ below the bifurcation (three measurements on each side), and the average measurement was taken as the IMT. IMT measurements were always performed in plaque-free arterial segments.

\section{Evaluation of common femoral artery IMT}

Ultrasonographic studies on common femoral arteries were performed bilaterally by a single observer. The value of common femoral arteries that was considered for statistical analyses was the mean of right and left measurements. All studies were performed with a Hewlett Packard Sonos 1500 (Hewlett Packard) using a 7.5 MHz high-resolution probe. IMT was defined as a low-level echo grey band that does not project into the arterial lumen. It was measured during enddiastole as the distance from the leading edge of the second echogenic line of the far walls of the distal segment of the common femoral artery on both sides. Measurements were performed $0.5,1$, and $2 \mathrm{~cm}$ below the femoral (profunda and superficial; three measurements on each side), and the average measurement was taken as the IMT.

\section{Evaluation of infrarenal anteroposterior diameter of abdominal aorta}

Ultrasonographic studies on infrarenal abdominal aorta was performed ${ }^{18-20}$ by a single observer. All studies were performed with a Hewlett Packard Sonos 1500 (Hewlett Packard) using a $3 \mathrm{MHz}$ high-resolution probe. All patients fasted for six hours prior to the ultrasonography. With the patient in a supine position, the electronic probe was placed one centimeter left of the umbilicus. The best image in longaxis projection of the abdominal aorta was then obtained. The anteroposterior diameter of the aorta was defined as the maximal external cross-sectional measurement. It was calculated as the distance between the near and the far walls of the abdominal aorta. Measurements were performed at $0.5,1$, and $2 \mathrm{~cm}$ above the umbilicus and were expressed in centimeters. In our study, the abdominal aorta was nonaneurysmatic in all women. 
Table I General, anthropometric, and metabolic parameters in PCOS and control women

\begin{tabular}{llll}
\hline & PCOS $(\mathbf{N}=\mathbf{2 9})$ & Control $(\mathbf{N}=\mathbf{2 6})$ & Statistical significance \\
\hline Age (years) & $22 \pm 3.8$ & $22 \pm 3.8$ & NS \\
Body mass index $\left(\mathrm{kg} / \mathrm{m}^{2}\right)$ & $26.3 \pm 4.5$ & $20.5 \pm 1.6$ & $\mathrm{P}<0.00 \mathrm{I}$ \\
Waist-to-hip ratio & $0.86 \pm 0.05$ & $0.71 \pm 0.05$ & $\mathrm{P}<0.00 \mathrm{I}$ \\
Systolic blood pressure $(\mathrm{mm} \mathrm{Hg})$ & $112.6 \pm 7.8$ & $107.9 \pm 9$ & $\mathrm{P}$ \\
Diastolic blood pressure $(\mathrm{mm} \mathrm{Hg})$ & $72.1 \pm 8.5$ & $66.9 \pm 8.6$ & $\mathrm{P}<0.05$ \\
Fasting insulin $(\mu \mathrm{Ul} / \mathrm{ml})$ & $16.6 \pm 3.9$ & $11.6 \pm 2.9$ & $\mathrm{P}<0.00 \mathrm{I}$ \\
Fasting blood glucose $(\mathrm{mg} / \mathrm{dl})$ & $84.95+9.74$ & $82.98+5.83$ & $\mathrm{NS}$ \\
Total cholesterol $(\mathrm{mg} / \mathrm{dl})$ & $187.8 \pm 3.6$ & $160.6 \pm 2.3$ & $\mathrm{P}<0.00 \mathrm{I}$ \\
HDL-C $(\mathrm{mg} / \mathrm{dl})$ & $53.4 \pm 14$ & $59.6 \pm 12$ & $\mathrm{NS}$ \\
LDL-C $(\mathrm{mg} / \mathrm{dl})$ & $116.4 \pm 3.1$ & $106.7 \pm 2.5$ & $\mathrm{P}<0.00 \mathrm{I}$ \\
Triglycerides $(\mathrm{mg} / \mathrm{dl})$ & $94.5 \pm 6.3$ & $65 \pm 2.9$ & $\mathrm{P}<0.05$ \\
Apo Al $(\mathrm{mg} / \mathrm{dl})$ & $133.2 \pm 16$ & $134.9 \pm 22$ & $\mathrm{NS}$ \\
Apo B $(\mathrm{mg} / \mathrm{dl})$ & $89.7 \pm 2.5$ & $73 \pm 2.05$ & $\mathrm{P}<0.00 \mathrm{I}$ \\
\hline
\end{tabular}

Abbreviations: Apo, apolipoprotein; HDL-C, high-density lipoprotein cholesterol; LDL-C, low-density lipoprotein cholesterol; NS, not significant; PCOS, polycystic ovary syndrome.

\section{Repeatability and intraobserver variability of ultrasound scan measurements}

Repeatability and intraobserver variability of all ultrasound scan measurements were evaluated by means of intraclass correlation coefficient of $>0.90$. In particular, anteroposterior diameter showed good reproducibility with an intraclass correlation coefficient of 0.92 .

\section{Statistical methods}

Results are presented as mean and standard deviation (SD) for all parameters. Variables with a skewed distribution (insulin, triglycerides, etc) were logarithmically transformed before any statistical analyses to improve the approximation to a Gaussian distribution. Differences between women with PCOS and the control group were performed by an unpaired Student $t$-test. Analysis of covariance was performed and corrected for other clinical variables. $P$ values of $<0.05$ were considered statistically significant. Statistical analyses were performed by using STATISTICA 6.1 software (StatSoft Inc., Tulsa, OK, USA).

\section{Results}

General, anthropometric, hormone, and metabolic characteristics of women with PCOS and control subjects are reported in Table 1. The groups were of similar age and all women were without any previous hormonal treatment, but differed significantly in most of the other parameters.

Compared to control women, women with PCOS had higher BMI, WHR, systolic and diastolic blood pressure levels, and higher fasting insulin, total and LDL-cholesterol, apo B, and triglyceride levels. HDL and apo A1 showed a tendency to be lower in women with PCOS. Sex hormones, $\mathrm{LH}$, testosterone, and androstenedione levels and LH to FSH

Table 2 Sex hormones in PCOS and control women

\begin{tabular}{llll}
\hline & PCOS $(\mathbf{N}=\mathbf{2 9})$ & Control $(\mathbf{N}=\mathbf{2 6})$ & Statistical significance \\
\hline LH $(\mathrm{mUl} / \mathrm{ml})$ & $1 \mathrm{l} .7 \pm 4$ & $4.9 \pm 2.8$ & $\mathrm{P}<0.00 \mathrm{I}$ \\
$\mathrm{FSH}(\mathrm{mUl} / \mathrm{ml})$ & $5.2 \pm 2$ & $5.4 \pm 1.5$ & $\mathrm{NS}$ \\
LH to FSH ratio & $2.6 \pm 1.2$ & $1.1 \pm 0.7$ & $\mathrm{P}<0.01$ \\
Testosterone $(\mathrm{ng} / \mathrm{dl})$ & $68.3 \pm 11.9$ & $43.2 \pm 10.0$ & $\mathrm{P}<0.00 \mathrm{I}$ \\
Androstenedione $(\mathrm{ng} / \mathrm{ml})$ & $3.9 \pm 1.2$ & $2.1 \pm 0.9$ & $\mathrm{P}<0.00 \mathrm{I}$ \\
Estradiol $(\mathrm{pg} / \mathrm{ml})$ & $37.6 \pm 11.9$ & $40 \pm 8.1$ & $\mathrm{NS}$ \\
SHBG $(\mu \mathrm{g} / \mathrm{ml})$ & $1.7 \pm 0.6$ & $3.5 \pm 1.2$ & $\mathrm{P}<0.00 \mathrm{I}$ \\
\hline
\end{tabular}

Abbreviations: FSH, follicle-stimulating hormone; LH, serum luteinizing hormone; NS, not significant; SHBG, sex hormone-binding globulin. 
Table 3 Ultrasound results in PCOS and control women

\begin{tabular}{llll}
\hline & PCOS $(\mathbf{N}=\mathbf{2 9})$ & Control $(\mathbf{N}=\mathbf{2 6})$ & Statistical significance \\
\hline IMTC $(\mathrm{mm})$ & $0.651 \pm 0.59$ & $0.637 \pm 0.133$ & NS \\
IMTF $(\mathrm{mm})$ & $0.655 \pm 0.168$ & $0.621 \pm 0.10$ & NS \\
APD $(\mathrm{mm})$ & $13.87 \pm 1.8$ & $12.18 \pm 2.3$ & $\mathrm{P}<0.01$ \\
\hline
\end{tabular}

Abbreviations: APD, anteroposterior diameter of abdominal aorta artery; IMTC, intima-media thickness of common carotid artery; IMTF, intima-media thickness of common femoral artery; NS, not significant.

ratio were higher in women with PCOS, who showed lower SHBG levels compared to control women (Table 2).

Vascular ultrasonographic parameters are reported in Table 3. Women with PCOS showed a significant higher anteroposterior diameter of the abdominal aorta compared to control women, and a tendency to an increase in carotid and femoral IMT. This result was confirmed $(p=0.024)$ by multivariate analysis of covariance correcting for systolic and diastolic blood pressure and WHR. When BMI was added as covariate, the comparison lacked statistical significance due to the large difference in BMI between the control and PCOS groups.

\section{Discussion}

The most original result of the present study is that the anteroposterior diameter of the abdominal aorta is significantly higher in women with PCOS than in normal weight and fertile women.

It has been recently shown that age, ${ }^{21} \mathrm{BMI},{ }^{21}$ and intraabdominal fat (measured by ultrasound) ${ }^{22}$ are significantly associated with increasing aortic diameter independent of other cardiovascular disease risk factors. Since PCOS and control women were similar in age in this study, whereas BMI and WHR were higher in women with PCOS, it may well be that BMI and abdominal fat are responsible for the higher anteroposterior diameter of the abdominal aorta in women with PCOS. In fact, anteroposterior diameter did not maintain a significant relationship when BMI was considered as covariate during multivariate analysis of covariance.

This study confirms that women with PCOS are at higher cardiovascular risk compared with control women, but it does not confirm the previously shown significant increase of common carotid artery IMT in women with PCOS. ${ }^{15-17}$ It is possible that the limited number of patients in our study may be responsible for the lack of a significant difference in this parameter. However, it is noteworthy that this study examined young PCOS women, 17 to 27 years old, with a mean age of 22 years. Since most previous studies investigating the relationship between PCOS and cardiovascular risk examined women with a mean age around 40 years, ${ }^{15-17}$ it may well be that the age of women in our study was too low to show artery thickening and differences between the groups. This may explain the lack of a significant difference in the common femoral artery IMT between women with and without PCOS.

Our findings also show that the increase of the anteroposterior diameter of the abdominal aorta is the earliest arterial alteration in women with PCOS, thus preceding the IMT of other arteries such as common carotid arteries and common femoral arteries. These results are in line with a recent paper by Allison and colleagues. ${ }^{21}$

According to previous studies, BMI, abdominal fat accumulation, insulin, total cholesterol, LDL cholesterol, triglyceride, and apo B levels were higher in PCOS patients compared to normal weight women. ${ }^{6-14}$

\section{Conclusion}

Anteroposterior diameter of the infrarenal abdominal aorta, but not IMT of common carotid arteries or common femoral arteries, is higher in patients with PCOS than in normal weight and fertile women, thus preceding the thickening of other arteries. However, this alteration seems to be due to body weight secondary to PCOS and not due to PCOS perse.

\section{Acknowledgments}

The authors wish to thank Dr. Pietro Guida for his assistance in the statistical analysis. The authors report no conflicts of interest in this work.

\section{References}

1. Rotterdam ESHRE/ASRM-Sponsored PCOS Consensus Workshop Group. Revised 2003 consensus on diagnostic criteria and long-term health risks related to polycystic ovary syndrome. Fertil Steril. 2004;81(1):19-25.

2. Azziz R, Goodarzi MO. Diagnosis, epidemiology, and genetics of the polycystic ovary syndrome. Best Pract Res Clin Endocrinol Metab. 2006;20(2):193-205.

3. Chang PL, Lindheim SR, Lowre C, et al. Normal ovulatory women with polycystic ovaries have hyperandrogenic pituitary-ovarian responses to gonadotropin-releasing hormone-agonist testing. J Clin Endocrinol Metab. 2000;85(3):995-1000. 
4. Azziz R, Black V, Hines GA, Fox LM, Boots LR. Adrenal androgen excess in the polycystic ovary syndrome: sensitivity and responsivity of the hypothalamic-pituitary-adrenal axis. J Clin Endocrinol Metab. 1998;83(7):2317-2323.

5. Cho LW, Randeva HS, Atkin SL. Cardiometabolic aspects of polycystic ovarian syndrome. Vasc Health Risk Manage. 2007;3(1):55-63.

6. Talbott EO, Guzick DS, Clerici A, et al. Coronary heart disease risk factors in women with polycystic ovary syndrome. Arterioscler Thromb Vasc Biol. 1995;15(7):821-826.

7. Meyer C, McGrath BP, Teede HJ. Overweight women with polycystic ovary syndrome have evidence of subclinical cardiovascular disease. J Clin Endocrinol Metab. 2005;90(10):5711-5716.

8. Berneis K, Rizzo M, Lazzaroni V, Fruzzetti F, Carmina E. Atherogenic lipoprotein phenotype and low-density lipoproteins size and subclasses in women with polycystic ovary syndrome. J Clin Endocrinol Metab. 2007;92(1):186-189.

9. Battaglia C, Mancini F, Cianciosi A, et al. Vascular risk in young women with polycycstic ovary syndrome. Obstet Gynecol. 2008;111(2 Pt 1):385-395.

10. Arslanian SA, Lewy VD, Danadian K. Glucose intolerance in obese adolescents with polycystic ovary syndrome: roles of insulin resistance and $\beta$-cell dysfunction and risk of cardiovascular disease. J Clin Endocrinol Metab. 2001;86(1):66-71.

11. Cheung LP, Ma RCW, Lam PM, et al. Cardiovascular risks and metabolic syndrome in Hong Kong Chinese women with polycystic ovary syndrome. Hum Reprod. 2008;23(6):1431-1438.

12. Shaw LJ, Bairey Merz CN, Azziz R, et al. Postmenopausal women with a history of irregular menses and elevated androgen measurements at high risk for worsening cardiovascular event-free survival: results from the National Institutes of Health - National Heart, Lung, and Blood Institute sponsored Women's Ischemia Syndrome Evaluation. J Clin Endocrinol Metab. 2008;93(4):1276-1284.

13. Tikkanen MJ, Nikkilä EA. Regulation of hepatic lipase and serum lipoproteins by sex steroids. Am Heart J. 1987;113(2 Pt 2):562-567.
14. Graf M, Richards CJ, Brown V, et al. The independent effects of hypeandrogenemia, hyperinsulinemia and obesity on lipid and lipoprotein profiles in women. Clin Endocrinol (Oxf). 1990;33(1): 119-131.

15. Talbott EO, Guzick DS, Sutton-Tyrrell K, et al. Evidence for association between polycystic ovary syndrome and premature carotid atherosclerosis in middle-aged women. Arterioscler Thromb Vasc Biol. 2000;20(11):2414-2421.

16. Talbott EO, Zborowski JV, Boudreaux MY, McHugh-Pemu KP, Sutton-Tyrrell K, Guzick DS. The relationship between C-reactive protein and carotid intima-media wall thickness in middle-aged women with polycystic ovary syndrome. J Clin Endocrinol Metab. 2004;89(12):6061-6067.

17. Vryonidou A, Papatheodorou A, Tavridou A, et al. Association of hyperandrogenemic and metabolic phenotype with carotid intima-media thickness in young women with polycystic ovary syndrome. J Clin Endocrinol Metab. 2005;90(5):2740-2746.

18. Wilmink AB, Forshaw M, Quick CR, Hubbard CS, Day NE. Accuracy of serial screening for abdominal aortic aneurysms by ultrasound. J Med Screen. 2002;9(3):125-127.

19. Lederle FA, Walker JM, Reinke DB. Selective screening for abdominal aortic aneurysms with physical examination and ultrasound. Arch Intern Med. 1988;148(8):1753-1756.

20. Brady AR, Gerald F, Fowkes R, Thompson SG, Powell JT. Aortic aneurysm diameter and risk of cardiovascular mortality. Arterioscler Thromb Vasc Biol. 2001;21(7):1203-1207.

21. Allison MA, Kwan K, DiTomasso D, Wright CM, Criqui MH. The epidemiology of abdominal aortic diameter. J Vasc Surg. 2008;48(1): 121-127.

22. Gorter PM, Vusseren FL, Moll FL, van der Graaf Y; SMART Study Group. Abdominal fat and metabolic syndrome are associated with larger infrarenal aortic diameters in patients with clinically evident arterial disease. J Vasc Surg. 2008;48(1):114-120.
Vascular Health and Risk Management

\section{Publish your work in this journal}

Vascular Health and Risk Management is an international, peerreviewed journal of therapeutics and risk management, focusing on concise rapid reporting of clinical studies on the processes involved in the maintenance of vascular health; the monitoring, prevention and treatment of vascular disease and its sequelae; and the involvement of

\section{Dovepress}

metabolic disorders, particularly diabetes. This journal is indexed on PubMed Central and MedLine. The manuscript management system is completely online and includes a very quick and fair peer-review system, which is all easy to use. Visit http://www.dovepress.com/ testimonials.php to read real quotes from published authors. 